\title{
Improved walking ability with wearable robot-assisted training in patients suffering chronic stroke ${ }^{1}$
}

\author{
Lifang $\mathrm{Li}^{\mathrm{a}, \mathrm{b}}$, Li Ding ${ }^{\mathrm{c}}$, Na Chen ${ }^{\mathrm{a}, \mathrm{b}}$, Yurong Mao ${ }^{\mathrm{a}, \mathrm{b}}$, Dongfeng Huang ${ }^{\mathrm{a}, \mathrm{b}}$ and Le Li ${ }^{\mathrm{a}, \mathrm{b},{ }^{*}}$ \\ ${ }^{a}$ Department of Rehabilitation Medicine, First Affiliated Hospital, Sun Yat-Sen University, \\ Guangzhou, 510080, China \\ ${ }^{b}$ Guangdong Provincial Research Center for Rehabilitation Medicine and Translational Technology, \\ Guangzhou, 510080, China \\ ${ }^{c}$ Department of Rehabilitation, Huashan Hospital, Fudan University, Shanghai, 200040, China
}

\begin{abstract}
Wearable robotic devices provide safe and intensive rehabilitation, enabling repeated motions for motor function recovery in stroke patients. The aim of this small case series was to demonstrate the training effects of a three-week robotic leg orthosis, and to investigate possible mechanisms of the sensory-motor alterations and improvements by using gait analysis and EMG. Three survivors of chronic strokes participated in robot-assisted gait therapy for three weeks. EMG signals from the rectus femoris (RF), tibialis anterior (TA), biceps femoris (BF), and medial gastrocnemius (MG), as well as kinetics and kinematics data of the lower limb, were recorded before and after the training. The normalized root mean squared (RMS) values of the muscles, the joint moments, joint angles, and the results of two clinical scales (Berg Balance scale, BBS, and the lower extremity subscale of Fugl-Meyer assessment, LE-FMA) were used for analysis. All participants experienced improved balance and functional performances and increased BBS and LE-FMA scores. The EMG results showed there was an increase of the normalized RMS values of the MG and BF on the affected side. Additionally, EMG activities of the agonist and antagonist pair (i.e. RF and BF) appeared to return to similar levels after training. The peak moment of hip flexor, knee extensor, and plantar flexor, which all contributed to push-off power, were found to have increased after training. In summary, the three-week training period using the wearable RLO improved the three participants' gait performance by regaining push-off power and improved muscle activation and walking speed.
\end{abstract}

Keywords: Chronic stroke, robotic leg orthosis, gait performance, EMG

\section{Introduction}

Many ambulatory stroke survivors suffer alterations to their gait patterns as a result of the stroke. Unfortunately, in a majority of patients, self exercises without professional assistance in the chronic stages can result in the development of stubborn abnormal gait patterns, including stiff-knee gait

\footnotetext{
${ }^{1}$ Lifang Li and Li Ding contributed equally to this work.

*Address for correspondence: Le Li, Department of Rehabilitation Medicine, First Affiliated Hospital, Sun Yat-Sen University, Guangzhou, 510080, China. Tel.: +86-20-87332200-8536; Fax: +86-20-87750632; E-mail: lile5@mail.sysu.edu.cn.
}

0959-2989/15/\$35.00 @ 2015 - IOS Press and the authors. 
(SKG) [1], limited or absent ankle dorsiflexion, impairment of loading ability on the paretic side [2], and abnormal or insufficient muscle activation or contraction $[3,4]$, which can greatly influence their quality of life. Experience-dependent plasticity, regarded as a potential mechanism of the neural networks, allows stroke survivors to recover motor function control and partially offset impairments $[5,6]$.

Recent technologies have made it possible for robotic devices to provide safe and intensive rehabilitation therapy using repeated motions. Studies have shown that robotic leg orthosis (RLO) can improve patients' gait and walking ability [7-9]. However, few researches have reported the effect of the robot-assisted training on the improvements of gait performance which integrated 3D motion gait analysis and the muscle activation analysis. In addition, little is known regarding the underlying mechanisms of gait alternations after RLO training.

In this case study, gait performance changes were investigated in three chronic stroke patients who received robot-assisted gait therapy (a new wearable robotic orthosis) at two speeds (self-selected and maximal). An attempt was made to explore the training effects and possible mechanisms of sensory-motor changes using gait and EMG analysis after three weeks of training.

\section{Methods}

\subsection{Participants}

Three stroke survivors were recruited from the Department of Rehabilitation Medicine, First Affiliated Hospital, Sun Yat-sen University. All subjects had suffered one stroke and could actively move their legs and walk more than $10 \mathrm{~m}$ without a walking aid when first examined for this study. All participants were without other neurologic disorders, uncontrolled hypertension, cognitive deficits (MMSE<23), or other internal diseases that prevented their participation. Table 1 summarizes the characteristics of the participants, with additional narrative below.

Before the rehabilitation protocol, each participant had one to two days to become familiar with the basic functions of the RLO. The participants were taught the basic design of the RLO by a physical therapist, and were then allowed to walk with the RLO independently.

Table 1

Participant's characteristics

\begin{tabular}{|c|c|c|c|}
\hline & Participant 1 & Participant2 & Participant 3 \\
\hline Sex & $\mathrm{M}$ & $F$ & $\mathrm{~F}$ \\
\hline Age & 62 & 61 & 53 \\
\hline Height $(\mathrm{mm})$ & 1650 & 1590 & 1580 \\
\hline Weight $(\mathrm{kg})$ & 72 & 55 & 60 \\
\hline Stroke type & Ischaemic & Ischaemic & Hemorrhagic \\
\hline Hemiplegic side & Left & Right & Right \\
\hline Lesion position & $\begin{array}{l}\text { Without imaging } \\
\text { report }\end{array}$ & $\begin{array}{l}\text { Ischemic area in white matter of left } \\
\text { paraventricular (the maximum level is } \\
\text { about } 13 \times 22 \mathrm{~mm} \text { ) }\end{array}$ & $\begin{array}{l}\text { Basal ganglia } \\
\text { hemorrhagic stroke, } \\
\text { with } 35 \mathrm{ml} \text { bleeding } \\
\text { volume }\end{array}$ \\
\hline $\begin{array}{l}\text { Spasticity of knee extensors } \\
\text { (Modified Ashworth Scale) }\end{array}$ & $1+$ & 1 & 2 \\
\hline Time since stroke(months) & 59 & 25 & 8 \\
\hline
\end{tabular}




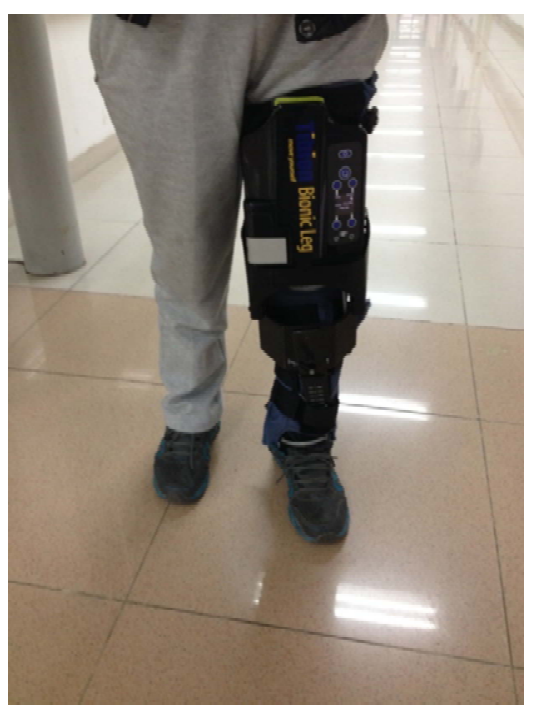

Fig. 1. Participant wearing Tibion Bionic Leg (Tibion Corporation, Sunnyvale, CA, USA).

\subsection{Intervention and training procedures}

The three-week robot-assisted physical therapy training program involved the participants utilizing a wearable RLO, an active exoskeleton Tibion Bionic Leg (Tibion Corporation, Sunnyvale, CA, USA), as shown in Figure 1. The device had a weight of 3.5 kilograms and an internal sensor that detected the wearer's foot pressure. The RLO was activated and a provided forward propulsion when the participants generated enough force on their paretic knee. The RLO provided assistance with extension (during stance phase, forward walking, and so on), controlled flexion (during stand-to-sit), and free movement (during the swing phase) [10]. Three settings on the device could be changed: threshold (the minimum force to activate the device), assistance (the percentage of body weight provided through the limb during extension in the stance phase of the gait cycle), and resistance (level of resistance during flexion on the stance phase of the gait cycle).

The robot-assisted physical therapy training protocol was designed following a motor control learning theorem that included fifteen sessions conducted five times per week for three weeks. Each one-hour session included approximately 50 minutes of training with the RLO and brief rest periods of no more than 10 minutes, as needed.

The RLO training program included sit-to-stand transfers; static and dynamic standing balance; daily walking in the clinics at various speeds; moving backward or laterally, moving up and down stairs; and mobility around obstacles. A physical therapist accompanied the participant sat all times during training sessions to avoid the risk of falling. As each participant's abilities changed, the device

Table 2

Parameter alterations before and after training

\begin{tabular}{llll}
\hline & Threshold (\%) & Assistance (\%) & Resistance \\
\hline P 1 & $60 / 80$ & $30 / 30$ & high \\
P 2 & $30 / 70$ & $70 / 30$ & medium \\
P 3 & $30 / 60$ & $70 / 35$ & medium \\
\hline
\end{tabular}

Notes: P1-3 means participant 1 to participant 3. Data with slash means the parameter setting in the first week and the last week of the training. 
parameters and training activities were progressively modified (Table 2). Due to the orthosis' changeable settings, the effects of the training could be analyzed in detail. All training protocol using the robotic leg was performed in the First Affiliated Hospital, Sun Yat-sen University with full consent of the participants.

After the three-week rehabilitation period, the use of the RLO was discontinued and all assessments were performed without the device. Gait analysis and clinical tests were conducted one day before the training and one day after the three weeks of training by the same researchers in the Motor Recovery Research Laboratory.

\subsection{Clinical tests}

Berg balance scale (BBS) and lower extremity subscale of Fugl-Meyer assessment (LE-FMA) were used in this study. BBS is a 14-item, 56-point scale designed to measure balance in older people, or patients with balance disorders, using assessments of functional tasks [11]. It is an effective assessment tool for use in clinical practice [12]. FMA consists of two motor subscales for measuring upper and lower extremity (i.e. UE and LE) movements [13]. Each item is scored on a 3-pointscale, from 0 to 2.LE-FMA has a score range of 0-34. LE-FMA has been shown to be a reliable and valid tool for LE functional assessment of stroke patients $[8,9]$. It consists of 17 items that measured in the supine, prone, sitting, and standing positions.

\subsection{EMG acquisition and data analysis}

The EMG signals were amplified by a wireless EMG (Noraxon, Telemyo DTS desk receiver, USA), with 8 channels (sampling frequency $=1,000 \mathrm{~Hz}$ ). Surface electrodes were attached torectus femoris (RF), tibialis anterior (TA), biceps femoris (BF), and gastrocnemius medial head (GM) bilaterally based on SENIAM [14]: The EMG signals were processed offline using MR-XP Master Edition (version 1.08). Band-pass filtering was applied to the EMG signals (from 10 to $500 \mathrm{~Hz}$ ). The root mean squared (RMS) [15] values were calculated. Forthe study, participants were instructed to perform a maximal voluntary contraction (MVC) of knee flexion and extension, ankle plantarflexion, and dorsiflexion for 5 seconds, and the maximum value of the torque for this movement was set as $100 \%$ of the MVC contraction level. For evaluation purposes, three healthy senior citizens were tested for leg muscle activation at self-selected speeds to compare against the three study participants.

\subsection{Motor reconstruct system and gait data analysis}

The kinematic data of each participant's hemiplegic lower extremity were measured using the Vicon Motion Analysis System (VICON MX13, $100 \mathrm{~Hz}$, Oxford, UK). Six infrared $100 \mathrm{~Hz}$ cameras recorded the data, which were subsequently analyzed using Vicon Nexus(version 1.7.1) The kinetic data were collected by two force plates (AMTI, Watertown, MA, USA) with a sampling frequency of $1,000 \mathrm{~Hz}$ embedded in the walkway [16].

Before each round of analysis, the participant was asked to perform trials in a warm-up stage. During the tests, the participant was asked to walk back and forth at a comfortable self-selected speed ("You are going on a walk in the garden") and a maximal maintainable speed ("Hurry up, or you will miss the bus, but be careful).The lower extremity data were collected both before and after the training protocol. Then kinematic, kinetic, and EMG data were simultaneously collected for four gait cycles. The maximum and minimum values of the lower limb kinematic variables (sagittal plane, hip, 
Table 3

BBS and LE-FMA scores before and after training

\begin{tabular}{lccc}
\hline & Pre & Post & $\Delta$ \\
\hline Berg Bbalance Scale(BBS) & & & \\
P 1 & 49 & 51 & 2 \\
P 2 & 40 & 45 & 5 \\
P 3 & 44 & 48 & 4 \\
Lower Extremity Subscale of & & & \\
Fugl-Meyer Assessment & & & \\
(LE-FMA) & 23 & 27 & 4 \\
P 1 & 20 & 25 & 5 \\
P 2 & 11 & 16 & 5 \\
P 3 & & \\
\hline
\end{tabular}

knee, and ankle joint angle, and the spatiotemporal parameters), and kinetic variables (sagittal plane hip, knee, and ankle joint moment) were compared pre and post training.

All parameters were considered for both aspects of the affected and unaffected sides, walking at the self-selected and maximal speeds, and before and after training.

\section{Results}

\subsection{Clinical tests results}

Table 3 shows the results of BBS and LE-FMA pre- and post-RLO training. As can be seen, all participants showed positive changes in their clinical measures.

\subsection{EMG results}

Figure 2 shows the normalized RMS EMG on the affected side, i.e. (RMS when walking/ MVC) $\times 100 \%$; in a specific muscle, EMG segments were collected during the whole gait cycle, and the RMS value is based on average among the 4 gait cycle tested. There were increases of the normalized RMS values of the MGs and BFs, and decreases of the RFs at two different speeds.

\subsection{Gait analysis results}

Gait analysis of the three participants was conducted on the self-selected and maximum walking speeds. The alterations of the spatiotemporal parameters showed improvements of gait performance in cadence, step length, and walking speed (Table 4). Mean increases of hip flexor, knee extensor, and plantarflexor moment (maximal values) for the participants were also seen after the training (Table 5). The lower limb peak joint angle of the paretic leg on the sagittal plane were also analyzed, the results of which are shown in Table 6.

\section{Discussion}

Recently, there has been a growing interest in using robots for the rehabilitation of patients who 
P 1
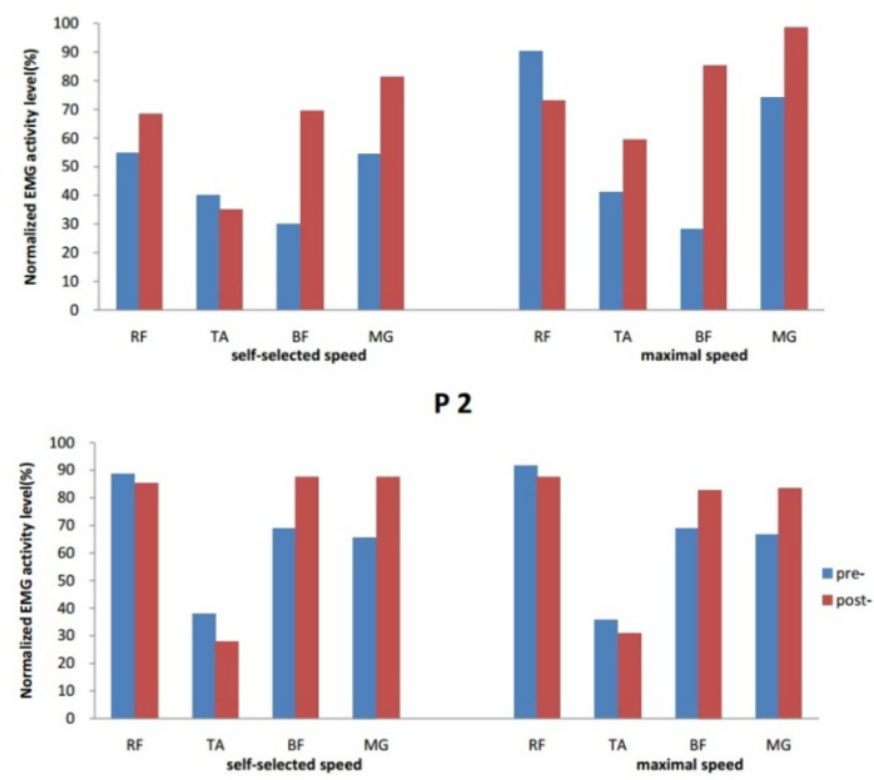

P 3

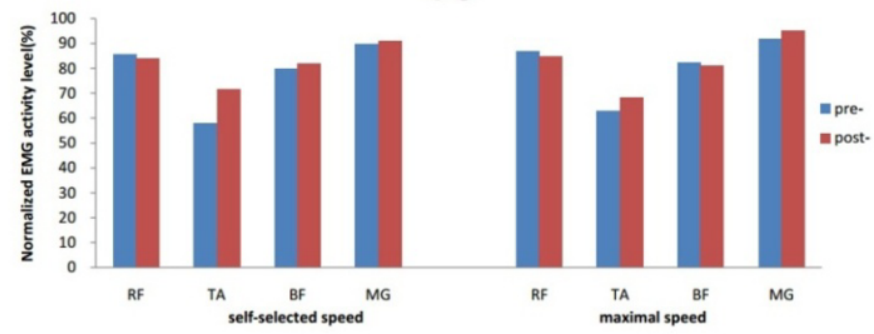

Fig. 2. Normalized RMS values (\%MVC) of RF, TA, BF, MG on affected side before and after training.

Table 4

Alterations of spatiotemporal parameters in each participant

\begin{tabular}{lccccccc}
\hline & & pre & post & $\Delta$ & pre & post & $\Delta$ \\
\hline Cadence(steps/min) & P1 & 104.59 & 112.12 & 7.53 & 118.81 & 120.25 & 1.45 \\
& P2 & 51.44 & 57.73 & 6.29 & 64.57 & 61.34 & -3.24 \\
& P3 & 66.82 & 64.37 & -2.44 & 68.03 & 65.93 & -2.10 \\
Stride Length(meters) & P1 & 0.78 & 0.89 & 0.11 & 0.84 & 1.04 & 0.19 \\
& P2 & 0.43 & 0.50 & 0.07 & 0.29 & 0.50 & 0.21 \\
& P3 & 0.47 & 0.49 & 0.02 & 0.49 & 0.52 & 0.03 \\
Step Length(meters) & P1 & 0.39 & 0.43 & 0.04 & 0.40 & 0.49 & 0.09 \\
& P2 & 0.21 & 0.26 & 0.05 & 0.16 & 0.27 & 0.11 \\
& P3 & 0.28 & 0.29 & 0.01 & 0.30 & 0.29 & -0.01 \\
Walking Speed(m/s) & P1 & 0.68 & 0.88 & 0.20 & 0.83 & 1.04 & 0.20 \\
& P2 & 0.14 & 0.24 & 0.10 & 0.16 & 0.25 & 0.09 \\
& P3 & 0.26 & 0.27 & 0.01 & 0.28 & 0.29 & 0.01 \\
\hline
\end{tabular}


Table 5

Lower limb joint moment (maximal values) before and after training

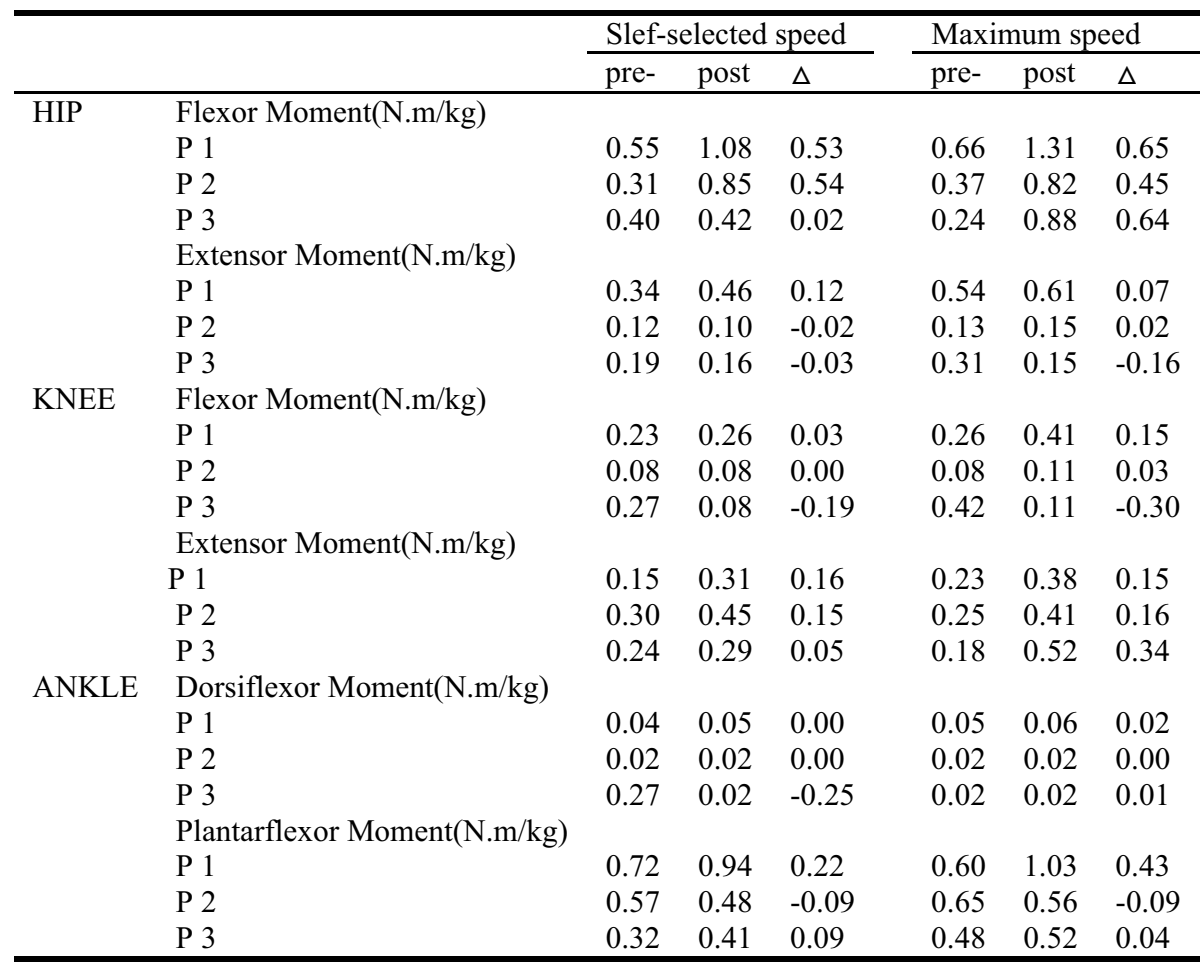

Table 6

Peak angle values (maximal values) of the hemiplegic side hip, knee, and ankle at different speeds before and after training

\begin{tabular}{lccccccc}
\hline & \multicolumn{3}{c}{ Slef-selected speed } & & \multicolumn{3}{c}{ Maximum speed } \\
\cline { 2 - 4 } \cline { 6 - 8 } & pre- & post & $\Delta$ & & pre- & post & $\Delta$ \\
\hline Peak Hip Flexion $\left(^{\circ}\right)$ & & & & & & \\
P 1 & 36.03 & 20.68 & -15.35 & & 33.82 & 22.25 & -11.57 \\
P 2 & 34.30 & 20.87 & -13.43 & & 29.05 & 25.79 & -3.26 \\
P 3 & 26.03 & 32.98 & 6.96 & & 29.32 & 33.10 & 3.78 \\
Peak Knee Flexion $\left({ }^{\circ}\right)$ & & & & & & & \\
P 1 & 48.45 & 49.35 & 0.90 & & 47.39 & 51.42 & 4.04 \\
P 2 & 24.32 & 25.07 & 0.75 & & 26.81 & 32.76 & 5.94 \\
P 3 & 22.83 & 13.77 & -9.06 & & 23.61 & 6.54 & -17.06 \\
Peak Ankle Plantarflexoin $\left({ }^{\circ}\right)$ & & & & & & \\
P 1 & 16.57 & 16.49 & -0.08 & & 15.87 & 15.57 & -0.30 \\
P 2 & 16.76 & 19.36 & 2.59 & & 16.42 & 18.64 & 2.22 \\
P 3 & 14.82 & 12.58 & -2.24 & & 18.50 & 14.55 & -3.94 \\
\hline
\end{tabular}

have suffered stroke [16]. Robot-assisted rehabilitation can deliver high-dosage and high-intensity training, making it helpful for stroke patients with motor disorders, especially those with gait problems. This case series study presented changes in clinical outcomes, EMG signals, and kinetic and kinematic parameters to demonstrate the effects of a new wearable robotic leg in chronic stroke patients, who participated in the three-week RLO training protocol. Gait performance improvements and muscle activation alterations in the three participants were discussed. 
After the training, all three participants demonstrated improvements without the use of the RLO on balance and lower extremity functional outcome measures. The improvements of balance might have been due to the unlimited functional activities in treatment, and the post-treatment BBS scores suggested a decreased fall risk [17]. The lightweight RLO in this study was used with stroke survivors who could actively move their legs and ambulate independently and who could take part in daily and functional activities without limitations. This might have encouraged more weight-bearing training and potentially stimulated more activities, which may have facilitated transference that benefitted their ADL and balance control. Additionally, the daily training involving stairs, obstacles, and transfers may have positively affected the participants' performance on the functional tests that included those activities. And because of the balance control and functional improvements, the cadence, stride length, and walking speeds showed an increasing trend, especially the walking speed at self-selected and maximum speed walking.

\section{1. $E M G$}

In this case series study, the EMG was applied in the test to calculate muscle activation. Increased neural drives can be reflected by increases in the EMG root mean squared (RMS), and the increasing neural stimulation from the neural system after a stroke will cause an obvious variation in EMG RMS [15]. Normalized RMS values were used in this study to reflect muscle activation intensity.

A closer examination of the EMG of participants before and after the training indicated that all the participants showed an increased intensity of MG and BF EMG activity at the self-selected speed and maximal speed on their paretic side, the activation of RF and BF of the three participants approached the same level, and the activation of MG was greater than TA after training, which suggested co-activation across the knee and ankle. The MG muscle with greater activation provided push-off power for the participants, but at the maximal speed, the activation of MG exhibited little increase. Schmitz, et al. believed that older adults depend less on plantarflexor muscle activation to push off at faster walking speeds because of alterations of their neuromuscular activity [18]. This may have been one of the reasons for the small increase of MG activation in the study.

It has been reported that increased activation of the adductor longus and soleus muscles is strongly associated with improved walking speeds in the first 6 months after stroke [19]. Therefore, the increased MG muscle activation should improve the push-off power of the ankle joint. For the rise of MG muscle activities, the internal sensor of the RLO and the positive biofeedback mechanism were important. The biofeedback treatment seemed to improve the neural activation patterns [20]. With this positive biofeedback, patients may be more willing to use their plantarflexors to move. The increased EMG intensity of BF may be the result of backward walking training, which requires hip extension and knee flexion. During walking, the hamstring muscles function primarily as hip extensor muscles, acting to decelerate the flexing hip from mid swing until initial contact, as well as to extend the hip during the first half of stance [21]. Participant 3 (P3) made only nine visits in the three-week training program, so no obvious changes in her muscle activations were seen as compared toP1 and P2.

Rybar, et al. reported that at maximal walking speed, a stroke patient often shows a lack of modulation in hip flexor muscle activation because of their lowered ability to increase the neural stimulation from the paretic nervous system and lower motor unit synchronization [22, 23]. The small increase of normalized RMS values of RF at maximal speed with the hip flexion angle rise after the training period indicated that the participants had increased agonist muscle (RF) modulation, as they could regulate moderate muscle behavior and recruit more motor units to perform hip flexion and achieve maximal speed walking speed. 


\subsection{Gait Performance - kinetic parameters}

The kinetic data of the joint moment showed that the hip flexor moment, knee extensor moment, and the plantar flexor moment were enhanced after the RLO training, resulting in movement more similar to normal gait than before the training period [24].

This hip flexor moment improvement indicated that the symmetrical paretic step length and hemiplegic gait characterized by the external rotation and abduction of the hip might progress into a normal gait pattern to some extent. Allen, et al. [4] demonstrated that in a group of stroke patients, larger hip flexor moments on the paretic side helped to improve walking symmetry. Symmetrical walkers appeared to utilize a bilateral hip strategy, which uses the hip flexor muscle, to accelerate the leg forward during pre-swing and swing to overcome the plantarflexor weakness [25]. This may be a reasonable explanation for the improvements of hip flexor moments. Moreover, the weight of the wearable RLO and the stairs training may have encouraged hip flexor activities, leading to the changes.

Knee extensor and plantarflexor muscles are important muscle groups responsible for generating a large part of the energy required to move the lower limbs forward during the push-off phase [26, 27]. In the involved side of stroke patients, the moment and power produced by the plantarflexors are highly related to gait performance [27, 28]. In the present study, improvements of knee extensor and plantarflexor moments in the participants (except P2) were seen. Based on the biofeedback from the RLO, when the participant generated enough plantar flexion force on the insole, the assistance from the knee provided him with anterior propulsion. As the biofeedback showed, the sit-to-stand and up and down stairs training played an important role in the increase of knee extensor moments. The data showed there was no obvious change of plantar flexor moment inP2. In order to overcome insufficiencies, stroke patients reportedly utilize other body mechanisms to compensate for the weakness of their plantarflexors [29]. As such, P2 had an increase in paretic leg hip flexor output and knee extensor output. The increased hip flexor moment and the modestly increased knee extension moment were considered compensation for her plantarflexor weakness [4, 28, 29].

Decreased peak knee flexion is one of the most common kinematic gait abnormalities in stroke patients [30]. Furthermore, sufficient knee flexion is of great importance for toe clearance, which is related to falls [31]. There were no obvious changes of knee flexion angles after training for P2, and P3 showed a decrease, which was correlated with insufficient plantarflexor moment. As for P3, it was believed she used her pelvis to compensate for insufficient knee flexion and weakness of the hip flexor. Additionally, her decreased knee flexion may have been due to the increased hip flexion angle. Furthermore, wearing AFO when training may be one of the reasons for the decrease of the knee flexion and plantarflexor moment output. However, detailed analysis should be performed to argue this alteration for P3.

As gait speed increased, more strength was required of the lower limb muscles to propel the leg forward. The gait analysis results could be explained by compensatory strategies developed by the participants during walking at the maximal speed. P3 showed a large increase in her plantarflexor moment and knee extension output at maximal speed in order to generate sufficient impulsion. And her hip flexion angle also increased at this speed; but a decrease of knee flexion angle was observed, which was regarded as compensation of SKG. P1 showed increased kinematic and kinetic hip parameters at maximal speed. Obvious findings were the improvements of plantarflexor moments and decreased knee extension moments at maximal speed after the training. And the increasing of range of moment from self-selected speed to fast speed became small after training. P1 was thought to have a strong plantarflexor, which could diminish the knee extension output. For P2, however, the weakness 
of the plantarflexor required her to propel with help from the knee extensor, and her speed did not increase very much.

The length and weight of the RLO could limit users' hip flexion, especially among Asian wearers. And because of the repeated training and movement relearning, participants could develop a small hip flexion. It has also been suggested that a shortened hamstring muscle after fatigue can lead to a reduction of hip flexion angle [32]. In this study, the decrease of the hip flexion was seen as the result of the walking speed being increased, which diminished the time for hip elevating. After the three-week training period using the RLO, it was believed that the three participants' alterations of muscle activation, kinematics, and kinetics at their maximal walking speeds became more normal with the drop of compensation.

\section{Conclusion}

In recent years, robot-assisted therapy based on the concepts of repetitive, intensive, and task-oriented training has been developed. In this study, a wearable RLO was used to demonstrate the effects of RLO training on gait performance in chronic stroke patients. The results suggested that the participants showed balance and lower extremity function improvements and enhanced gait performance with increased hip flexor, knee extensor, and plantarflexor moments. With the help of the RLO training, the muscle activation of the MG increased. Additionally, there was better modulation and co-contraction of the lower limb muscles following the training.

This case-series suggests that using the RLO for task-oriented physical therapy and lower extremity training may have benefited the stroke survivors' gait performance and muscle activation. This type of robot-assisted therapy could constitute a novel rehabilitation approach to improve the gait of chronic hemiparetic patients. However, large randomized clinical trials are needed to solidify these conclusions.

\section{Acknowledgments}

This study was supported by the National Natural Science Foundation of China (No. 31100669), Science and Technology Planning Project of Guangdong Province, China (No. 2013b090500099), Guangzhou Research Collaborative Innovation Projects (No. 2014Y2-00507) and the National College Students Innovation Training Program (No. 201310558088).

\section{References}

[1] I. Campanini, A. Merlo and B. Damiano, A method to differentiate the causes of stiff-knee gait in stroke patients, Gait Posture 38 (2013), 165-169.

[2] A. Mansfield, C.J. Danells, J.L. Zettel, S.E. Black and W.E. McIlroy, Determinants and consequences for standing balance of spontaneous weight-bearing on the paretic side among individuals with chronic stroke, Gait Posture 38 (2013), 428-432.

[3] N. Arene and J. Hidler, Understanding motor impairment in the paretic lower limb after a stroke: A review of the literature, Top Stroke Rehabilitation 16 (2009), 346-356.

[4] J.L. Allen, S.A. Kautz and R.R. Neptune, Step length asymmetry is representative of compensatory mechanisms used in post-stroke hemiparetic walking, Gait Posture 33 (2011), 538-543.

[5] T.H. Murphy and D. Corbett, Plasticity during stroke recovery: from synapse to behaviour, Nature Review 
Neuroscience 10 (2009), 861-872.

[6] T.H. Cruz, M.D. Lewek and Y.Y. Dhaher, Biomechanical impairments and gait adaptations post-stroke: Multi-factorial associations, Journal of Biomechanics 42 (2009), 1673-1677.

[7] R.W. Horst, A bio-robotic leg orthosis for rehabilitation and mobility enhancement, Conference Proceedings of IEEE Engineering and Medicine Biological Society, Minneapolis, USA, 2009, pp. 5030-5033.

[8] J. Sanford, J. Moreland, L.R. Swanson, P.W. Stratford and C. Gowland, Reliability of the Fugl-Meyer assessment for testing motor performance in patients following stroke, Physical Therapy 73 (1993), 447-454.

[9] P.W. Duncan, M. Propst and S.G. Nelson, Reliability of the Fugl-Meyer assessment of sensorimotor recovery following cerebrovascular accident, Physical Therapy 63 (1983), 1606-1610.

[10] N.N. Byl, Mobility training using a bionic knee orthosis in patients in a post-stroke chronic state: A case series, Journal of Medical Case Report 6 (2012), 216.

[11] K.O. Berg, S.L. Wood-Dauphinee, J.I. Williams and B. Maki, Measuring balance in the elderly: Validation of an instrument, Canadian Journal of Public Health 83 (1992), S7-S11.

[12] S.F. Tyson and L.A. Connell, How to measure balance in clinical practice: A systematic review of the psychometrics and clinical utility of measures of balance activity for neurological conditions, Clinical Rehabilitation 23 (2009), 824840.

[13] A.R. Fugl-Meyer, L. Jaasko and V. Norlin, The post-stroke hemiplegic patient, II. Incidence, mortality, and vocational return in Goteborg, Sweden with a review of the literature, Scandinavian Journal of Rehabilitation Medicine 7 (1975), 73-83.

[14] H.J. Hermens, B. Freriks, C. Disselhorst-Klug and G. Rau, Development of recommendations for SEMG sensors and sensor placement procedures, Journal of Electromyography Kinesiology 10 (2000), 361-374.

[15] X.L. Hu, K.Y. Tong and L. Li, The mechanomyography of persons after stroke during isometric voluntary contractions, Journal of Electromyography and Kinesiology 17 (2007), 473-483.

[16] W.H. Chang and Y.H. Kim, Robot-assisted therapy in stroke rehabilitation, Journal of Stroke 15 (2013), $174-181$.

[17] L. Alzayer, M. Beninato and L.G. Portney, The accuracy of individual Berg Balance Scale items compared with the total Berg score for classifying people with chronic stroke according to fall history, Journal of Neurologic Physical Therapy 33 (2009), 136-143.

[18] A. Schmitz, A. Silder, B. Heiderscheit, J. Mahoney and D.G. Thelen, Differences in lower-extremity muscular activation during walking between healthy older and young adults, Journal Electromyography Kinesiology 19 (2009), $1085-1091$.

[19] S. Mulroy, J. Gronley, W. Weiss, C. Newsam and J. Perry, Use of cluster analysis for gait pattern classification of patients in the early and late recovery phases following stroke, Gait Posture 18 (2003), 114-125.

[20] S.D. Din, A. Bertoldo and Z. Sawacha, Assessment of biofeedback rehabilitation in poststroke patients combining fMRI and gait analysis: A case study, Journal of Neuroengineering and Rehabilitation 11 (2014), 53.

[21] R.R. Neptune, K. Sasaki and S.A. Kautz, The effect of walking speed on muscle function and mechanical energetics, Gait Posture 28 (2008), 135-143.

[22] C.K. Thomas, J.E. Butler and I. Zijdewind, Patterns of pathological firing in human motor units, Advances in Experimental Medicine and Biology 508 (2002), 237-244.

[23] M.M. Rybar, E.R. Walker, H.R. Kuhnen, D.R. Ouellette, R. Berrios, S.K. Hunter and A.S. Hyngstrom, The stroke-related effects of hip flexion fatigue on over ground walking, Gait Posture 39 (2014), 1103-1108.

[24] J. Perry, Gait analysis: Technology and the clinician, Journal of Rehabilitation Research \& Development 31 (1994), vii.

[25] M.J. Mueller, D.R. Sinacore, S. Hoogstrate and L. Daly, Hip and ankle walking strategies: Effect on peak plantar pressures and implications for neuropathic ulceration, Archives of Physical Medicine and Rehabilitation 75 (1994), $1196-1200$.

[26] M. Thomas, Relative contributions of the lower extremity joint moments to forward progression and support during gait, Gait Posture 6 (1997), 1-9.

[27] S. Nadeau, D. Gravel, A.B. Arsenault and D. Bourbonnais, Plantarflexor weakness as a limiting factor of gait speed in stroke subjects and the compensating role of hip flexors, Clinical Biomechanics (Bristol, Avon) 14 (1999), $125-135$.

[28] C.K. Balasubramanian, M.G. Bowden, R.R. Neptune and S.A. Kautz, Relationship between step length asymmetry and walking performance in subjects with chronic hemiparesis, Archives of Physical Medicine and Rehabilitation 88 (2007), 43-49.

[29] R.E. Burke, A.M. Degtyarenko and E.S. Simon, Patterns of locomotor drive to motoneurons and last-order interneurons: Clues to the structure of the CPG, Journal of Neurophysiology 86 (2001), 447-462.

[30] S.J. Olney, M.P. Griffin and I.D. McBride, Temporal, kinematic, and kinetic variables related to gait speed in subjects with hemiplegia: A regression approach, Physical Therapy 74 (1994), 872-885.

[31] P. Levinger, D.T. Lai, H.B. Menz, A.D. Morrow, J.A. Feller, J.R. Bartlett, N.R. Bergman and R. Begg, Swing limb 
mechanics and minimum toe clearance in people with knee osteoarthritis, Gait Posture 35 (2012), 277-281.

[32] C. Chan, S. Yung and M. Liu, The change in lower limb kinematic and EMG activity of sprinting after soccer-specific fatigue among female soccer players, British Journal of Sports Medicine 48 (2014), 578. 